Document downloaded from:

http://hdl.handle.net/10251/66922

This paper must be cited as:

Borrás García, EM.; Tortajada-Genaro, LA.; Muñoz, A. (2016). Determination of reduced sulfur compounds in air samples for the monitoring of malodor caused by landfills. Talanta. 148:472-477. doi:10.1016/j.talanta.2015.11.021.

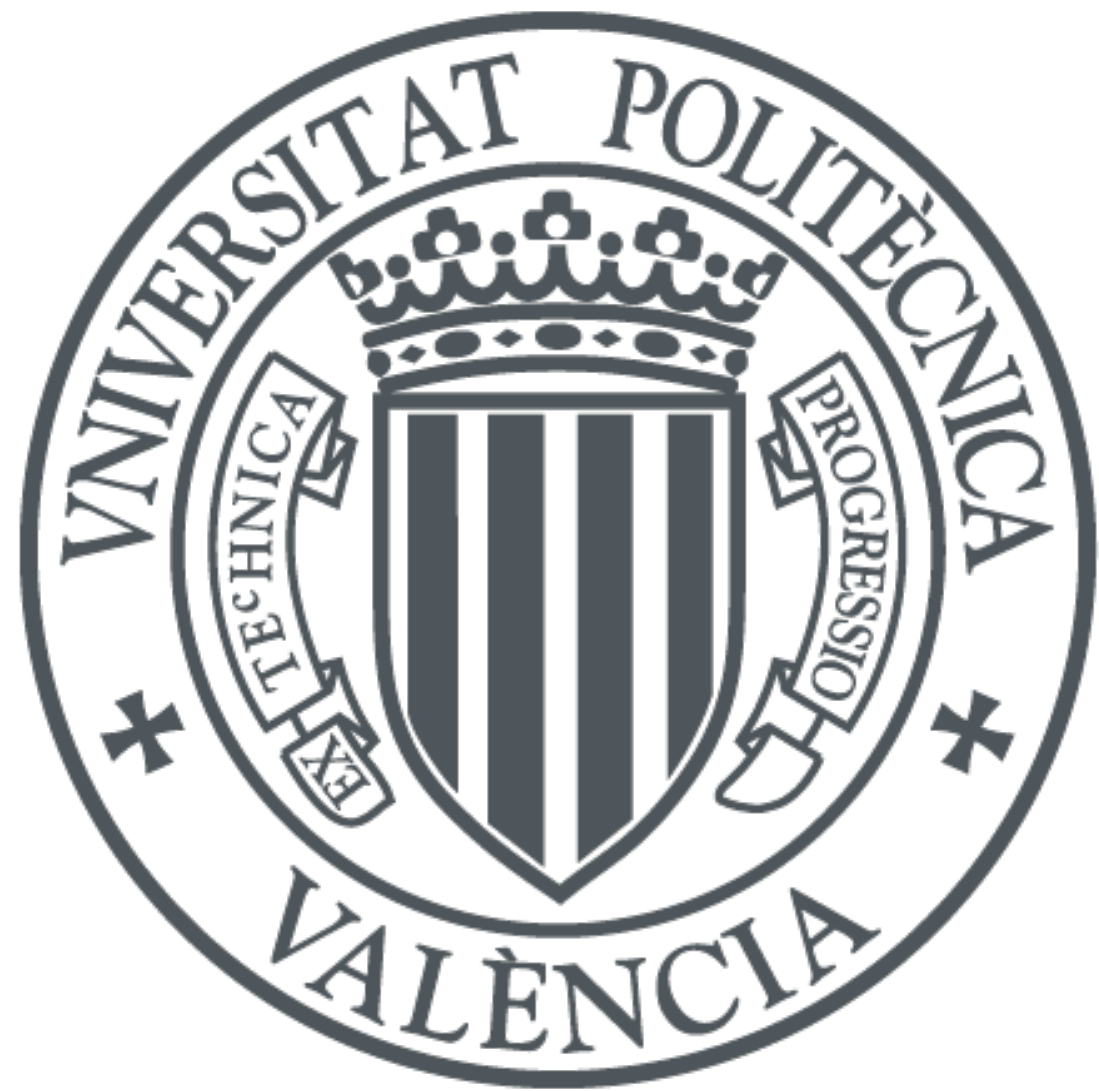

The final publication is available at

https://dx.doi.org/10.1016/j.talanta.2015.11.021

Copyright Elsevier

Additional Information 


\section{Determination of reduced sulfur compounds in air samples for the monitoring of malodor caused by landfills}

5 E. Borrás (1)*, L. A. Tortajada-Genaro (2), A. Muñoz (1)

1. Fundación Centro de Estudios Ambientales del Mediterráneo (CEAM), 46980 Paterna, Valencia, Spain.

2. Departamento de Química-Instituto IDM. Universitat Politècnica de València, 46022,

10 Valencia, Spain.

* Corresponding author:

15 Esther Borrás García

Instituto Universitario Centro de Estudios Ambientales del Mediterráneo CEAM - UMH (Fundación CEAM-UMH), 46980 Paterna, Valencia, Spain.

Phone: +34 961318227

Fax: +34 961318190

20 Email: esther@ceam.es 


\section{ABSTRACT}

A reliable method for determining malodorous reduced sulfur compounds (RSC) in atmospheric samples has been developed. The method uses an activated coconut solid-phase sorbent for active sampling, hexane as desorption solvent, and gas chromatography-mass spectrometry (GC-MS) technique for specific and sensitive separation-detection. The compounds analyzed were hydrogen sulfide, ethyl mercaptan, dimethyl sulfide, carbon disulfide, butyl mercaptan and dimethyl disulfide.

30 Recovery efficiency varied between $75-97 \%$ and no detectable losses were observed during storage at $-20{ }^{\circ} \mathrm{C}$. Satisfactory analytical parameters were reported, such as good linearity $\left(r^{2}>0.98\right)$, low detection limits $\left(0.6-59 \mathrm{pg} \mathrm{m}^{-3}\right)$, adequate repeatability (9 $\%)$ and reproducibility (17\%), and fast GC-MS analysis (<6.5 $\mathrm{min}$ ). The accurate determination of RSCs, free of interferences from atmospheric components, such as ozone or water was demonstrated. The method has been applied to analyze the composition of environmental air close to three landfills processing urban and industrial solid wastes. The results indicated that hydrogen sulfide and ethyl mercaptan were the main molecules responsible of malodor phenomenon in the study areas.

40 Keywords: malodor compounds, reduced sulfur compounds, solid-phase sampling, gas chromatography - mass spectrometry. 


\section{INTRODUCTION}

The presence of malodor species in the atmosphere causes a wide variety of social, environmental and health problems [1]. Odorous emissions are complex mixtures of pollutants directly discharged and by-products derived from biochemical reactions occurring under anaerobic conditions. Then, the sources of malodor are found to be including a variety of man-made activities such as chemical plants, oil refineries, sewage and wastewater treatment plants, landfills, livestock facilities, among others

$50[2,3]$. These emissions are mainly composed of volatile chemicals, such as alcohols, fatty acids, aldehydes, ketones, inorganic nitrogen derivates, organic and inorganic sulfur compounds [4,5]. Between them, reduced sulfur compounds (RSCs) have a relevant contribution, yielding very unpleasant odor perceivable by humans [2]. In fact, its presence in the atmosphere is a relevant nuisance to local populations [6]. Industrial sources of RSCs include fugitive emissions (leakages) from the steel industry, pulp and paper mills, refineries, incinerators, landfills, and sewage treatment facilities. Higgins et al. 2006 [7] studied the mechanisms for production and degradation of sulfur compounds, key molecules that cause odor produced by biosolids. Additionally, their chemistry in the atmosphere has significant implications for global climate changes by

60 impact radiative forcing, ozone layer depletion and the acidity of precipitation [8]. These observations accentuate the need for additional studies for odor mitigation and an increasing demand for methods for monitoring the emissions at the sources and at the influenced areas.

Intensive sampling campaigns and the recent developments in analytical

65 methodologies have contributed to improve the characterization of odorous emissions [1]. The major RSC released from malodor environments are hydrogen sulfide $\left(\mathrm{H}_{2} \mathrm{~S}\right)$, alkyl mercarptan (R-SH), dimethyl sulfide $\left(\mathrm{CH}_{3} \mathrm{SCH}_{3}\right)$, dimethyl disulfide $\left(\mathrm{CH}_{3} \mathrm{SSCH}_{3}\right)$ and carbon disulfide $\left(\mathrm{CS}_{2}\right)$ [2]. However, their determination in air samples is still a great challenge, e.g. their high reactivity leading to losses during sampling and 70 analysis.

Several systems have been studied for capturing RSCs, such as glass bulbs, canisters, Tedlar film bags, solid-phase micro-extraction, cryo-sampling, and cartridges [3,9-13]. Nevertheless, a revision of this stage is required to overcome drawbacks such as low efficiency, interferences due to atmospheric water, and artifacts associate to the matrix.

75 The use of scrubbers has been proposed to control and avoid the effect of oxidants, such as $\mathrm{SO}_{2}, \mathrm{O}_{3}$, humidity, and $\mathrm{NOx}$, during sampling [14]. However, they introduce more variability, reduce the sampling flow, and alter the caption/retention capacity of sampling surface. But, the most important restriction is the short storage time; for instance, samples collected by Tedlar bags must to be analyzed before $24 \mathrm{~h}$ or high 
losses have been reported [6]. Related to the extraction stage, thermal desorption is generally used due to its easy and reduced-time cost and its compatibility with several sampling strategies, but different limitations have been reported for the more volatile RSCs $[9,15]$. On the other hand, the use of organic solvent desorption present advantages such as very selective elution, usually highly efficient, applicable to a wide range of concentrations, and can be performed manually or automated with simple equipment. In addition, important efforts have sought the development of a fast, accurate method for quantifying captured RSCs present at very low concentrations. Gas chromatographic combined to mass spectrometry (GC-MS) methods, including a pre-concentration or derivatization step, are the most selected option $[2,12,16]$. Alternatively, electronic noses have been developed for a rapid measurement of malodor compounds, but the sensibility of these devices is quite reduced [17].

In a recent research, we have determined $\mathrm{CS}_{2}$ in air samples from pulp industry with excellent performances [18]. The goal of the present study was to extend this innovative approach by developing of a fast, sensitive, reproducible and efficient

95 fingerprint method, improving the current state of the art. The proposed approach is based on solid-phase sampling by activated charcoal surfaces, desorption with organic solvent, separation by gas chromatography without derivation, and detection by mass spectrometry. The studied RSCs included hydrogen sulfide, ethyl mercarptan, dimethyl sulfide, carbon disulfide, butyl mercaptan, and dimethyl disulfide. The physicalchemical information of six target malodor RSC compounds is shown in Table 1.

\section{EXPERIMENTAL}

\section{Reagents}

Hexane and toluene as HPLC grade solvent were purchased from Scharlau 105 (Barcelona, Spain). Hydrogen sulfide as gas state (>99\%), ethyl mercaptan, dimethyl sulfide, carbon disulfide, butyl mercaptan and dimethyl disulfide as liquid state (>99\%) were used supplied by Sigma Aldrich (Steinheim, Germany). Solid-phase support were Anasorb CSC coconut charcoal $(50 / 100 \mathrm{mg})$ combined with sodium sulfate dryers supplied by SKC limited (Dorset, UK), Orbo-32 activated coconut charcoal (100/50 mg) 110 (Sigma Aldrich, USA) and Tenax TEA (Sigma Aldrich, USA).

\section{Procedure}

Optimization experiments. Standard solutions of six RSC compounds in hexane (0.1-5 $\left.\mathrm{mg} \mathrm{L}^{-1}\right)$ were used for the GC-MS method development. Spiked air 115 samples, containing RSC selected compounds in an average concentration of $60 \mu \mathrm{g} \mathrm{m}^{-}$ 3 in pure air, were used for studying sampling and extraction step. Air mixtures were 
pumped through solid phase-cartridges based on activated coconut charcoal materials (total air volume $=0.24 \mathrm{~m}^{3}$ ). Three organic solvents - hexane, toluene and methanol, selected by their polarity and solubility properties - were studied for the liquid extraction using a low solvent consumption format $(0.5-5 \mathrm{~mL})$. Simultaneously, air mixtures were pumped through Tenax cartridges and RSC compounds were thermally desorbed by heating at $200^{\circ} \mathrm{C}$ during $5 \mathrm{~min}$ plus at $300^{\circ} \mathrm{C}$ during $5 \mathrm{~min}{ }^{\circ} \mathrm{C}$.

The retention yields and breakthrough volume were determined pumping gaseous RSC mixture through solid-phase sampling supports at $1 \mathrm{~L} \mathrm{~min}^{-1}$ (maximum sampling flow 125 allowed by technical cartridge properties) during different time periods ranged between $90 \mathrm{~min}$ and $72 \mathrm{~h}$, being the total air volume up to $4.3 \mathrm{~m}^{3}$. The effect of environmental interferences such as air humidity (0-60\%) and the presence of oxidant gaseous $\left(\mathrm{O}_{3}\right.$ $200 \mu \mathrm{g} \mathrm{m}^{-3}, \mathrm{NO}_{\times} 500 \mu \mathrm{g} \mathrm{m}^{-3}$ ) was also evaluated. For that, an ozonizator (UV-lamp) and NOx-calibration bottle were used, respectively.

130 The stability of the RSC malodor compounds stored on solid-phase cartridges was established by analyzing them immediately after the sampling, after storage during 1-5 days in closed dark bags at $4^{\circ} \mathrm{C}$, and after storage them during 12 weeks in closed dark bags at $-20^{\circ} \mathrm{C}$.

Finally, the standard addition method enable studying the presence of systematic

135 errors associated with matrix interferences. For that, a recovery study was performed, spiking different amounts of standard RSCs mixture (up to $0.150 \mu \mathrm{g}$ ) onto a ten solidphase cartridges sampled for $24 \mathrm{~h}$ at control ambient air.

Field sampling. Ambient air was collected at control locations and at landfill influenced areas in the southeastern of Spain (by duplicate). Ten samples from a sub140 urban locations (Control 1: 39 $35^{\prime} 05^{\prime \prime} \mathrm{N}, 0^{\circ} 27^{\prime} 42^{\prime \prime} \mathrm{W}$, Paterna, Valencia) and ten samples from a rural location (Control 2: 40³7'9"N, $0^{\circ} 6^{\prime} 2^{\prime \prime} \mathrm{W}$, Morella, Castellón) were used as air controls, free of direct malodor emission sources. Fifty-one air samples were collected at three influenced areas near to landfills (about $50 \mathrm{~m}$ distance). These landfills are used for waste management purposes of urban residues, being their 145 surface between 10 and 20 hectares. They process controlled solid waste, including not dangerous residues, construction waste, and industrial waste ( 3 - 10 tones year $\left.{ }^{-1}\right)$, electrical and electronic waste containing hazardous components (24 - 39 tones year $\left.{ }^{-1}\right)$ and non-hazardous waste $\left(<2000\right.$ tones year $\left.{ }^{-1}\right)$.

A low-volume pump (SIC Pocket Pump) connected to the solid phase cartridges 150 (sampling height: $1 \mathrm{~m}$ ) was used at flow of $1 \mathrm{~L} \mathrm{~min}^{-1}$. The samples were collected during $4 \mathrm{~h}$ (approximate $0.24 \mathrm{~m}^{3}$ ), at the same period (12:00 - 16:00 UMT) by during spring of 2014 (from $1^{\text {st }}$ to $30^{\text {th }}$ of May). The cartridges were stored at $4{ }^{\circ} \mathrm{C}$ until their analysis (before to $72 \mathrm{~h}$ ). 
Analysis of samples. Compounds retained on solid-phase supports were extracted dispensing $2 \mathrm{~mL}$ of hexane and sacking for $20 \mathrm{~min}$. For that, both solid-phase sorbent beds of activated coconut charcoal are transferred to a glass tubes and $2 \mathrm{~mL}$ of hexane are added. They are sacking at intervals of $5 \mathrm{~min}$ for a total of $20 \mathrm{~min}$, then, an aliquot of extract $(1 \mu \mathrm{L})$ was directly injected in the gas chromatograph - mass spectrometer. A TRACE-DSQ II instrument system was used (Thermo Fisher Scientific Co., Waltham, MA, USA) with a TRX-5MS column of $30 \mathrm{~m} \times 0.25 \mathrm{~mm}$ I.D $\times 0.25 \mu \mathrm{m}$ film thickness supplied by Thermo Fisher Scientific. Samples were injected in splitless

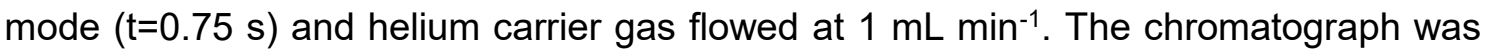
programmed at $32{ }^{\circ} \mathrm{C}$ for $2 \mathrm{~min}$, then ramped at a rate of $1^{\circ} \mathrm{C} \mathrm{min}^{-1}$ to $40{ }^{\circ} \mathrm{C}$, then ramped at a rate of $10^{\circ} \mathrm{C} \mathrm{min}-1$ to $160^{\circ} \mathrm{C}$ and held $1 \mathrm{~min}$. The injection port was held at $170{ }^{\circ} \mathrm{C}$ and the transfer line to the spectrometer was held at $300^{\circ} \mathrm{C}$. For detection, the ion source temperature was $200^{\circ} \mathrm{C}$ and the quadrupole temperature was $100{ }^{\circ} \mathrm{C}$. The impact electron ionization was operated at $70 \mathrm{eV}$ and the full select ion monitoring (SIM) acquisition mode was selected. The quantification was performed by the extraction of the base ion chromatographic peak of each compound (Table 1). The MS

170 did not acquire data while the solvent was eluting from the column (solvent delay: 2.51 - $3.2 \mathrm{~min})$.

The air quality at locations was classified based on the amount of total reduced sulfur (TRS), as the sum of concentrations of determined compounds. The categories, as function of thresholds, established by US Environmental Protection Agency (EPA) and European Environmental Agency (EEA) were: Very Good (No health effects are expected in healthy people) for 0-5 ppb; Good (Slight odor) for 6-10 ppb, Moderate (Odor) for 11-27 ppb, Poor (Strong odor) for 28-999 ppb, and Very Poor (Possible health effect) over $1000 \mathrm{ppb}$. Statistical package Statgraphics Centurion XVI for Windows was used for data analysis.

\section{RESULTS AND DISCUSSION}

\section{Chromatographic method}

The GC-MS variables were optimized for the simultaneous determination of targeted malodor compounds. Regarding chromatographic separation, previous studies for RSC determination used high apolar columns (e.g. DB-1) or long columns composed by diphenyl dimethyl polysiloxane $[5,15]$. In the present research, the selected column was $5 \mathrm{MS}$ column with a length of $30 \mathrm{~m}$, based on the better results obtained in our previous study for the single detection of $\mathrm{CS}_{2}$ [18]. This column showed a very low bleed and excellent inertness for active compounds, present in gaseous samples. A factorial design was performed to evaluate the influence of other chromatographic parameters. 
The standard solutions in hexane were injected in splitless and split mode (split ratios $1: 10,1: 20$, and 1:50) and separated changing working temperatures (oven programs) and carrier gas flow $\left(0.5\right.$ to $\left.1.5 \mathrm{~L} \mathrm{~min}^{-1}\right)$. These experiments indicated that more volatile RSC (hydrogen sulfide, ethyl mercaptan and dimethylsulfide) co-eluted under several conditions (resolution $R_{s}<0.5$ ). Nevertheless, an adequate separation was obtained injecting in splitless mode and separating at low initial temperature ( $32{ }^{\circ} \mathrm{C}$ for $2 \mathrm{~min}$ ) and intermediate He carrier flow $\left(1 \mathrm{~L} \mathrm{~min}^{-1}\right)$. Figure 1 shows a chromatogram under the selected conditions. The separation was reached in a total time of $8 \mathrm{~min}$, being the retention time of $1.70 \mathrm{~min}, 2.20 \mathrm{~min}, 2.31 \mathrm{~min}, 3.60 \mathrm{~min}, 6.18 \mathrm{~min}$ and, $7.72 \mathrm{~min}$ for $200 \mathrm{H}_{2} \mathrm{~S}, \mathrm{C}_{2} \mathrm{H}_{6} \mathrm{~S}, \mathrm{C}_{2} \mathrm{H}_{6} \mathrm{~S}, \mathrm{CS}_{2}, \mathrm{C}_{4} \mathrm{H}_{10} \mathrm{~S}$, and $\mathrm{C}_{2} \mathrm{H}_{6} \mathrm{~S}_{2}$, respectively. This elution order was in concordance with the polarity and molecular weight of such compound. The relative standard deviation of the retention times between replicated injections was lower than $1 \%$. The analysis time of the proposed method improved the previous ones described for detection of RSCs [12,19].

205 MS-detection parameters were evaluated to achieve a quantitative determination of studied RSCs. Detector gain $\left(1-10 \times 10^{5}\right)$, multiplier voltage (from 1100 to $2000 \mathrm{~V}$ ), filament emission current (from 50 to $200 \mu \mathrm{A}$ ), scan rate (from 0.3 to $1 \mathrm{scan} \mathrm{sec}^{-1}$ ) and mass defect (from 0 to 2 uma) were tested. The best results were obtained using $3 \mathrm{x}$

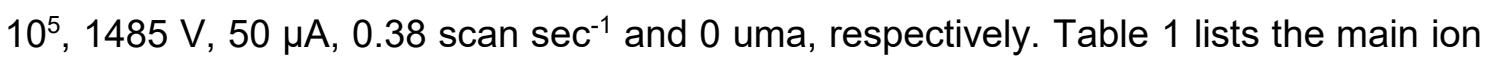

210 fragments and the base peak detected for each compound, coinciding with data from MS spectra registered in NIST database. Moreover, SCAN MS-acquisition mode and SIM MS-acquisition mode (selected ion monitoring) were checked. The SCAN mode (range from 50 to $300 \mathrm{~m} / \mathrm{z}$ and $0.95 \mathrm{~s}$ acquisition scan time) allowed assuring the correct identification of the detected RSC compounds. This option is especially 215 recommended for atmospheric monitoring in very complex air samples. Nevertheless, $\mathrm{SIM}$ mode, selecting the ion base $\mathrm{m} / \mathrm{z}$ fragment of each $\mathrm{RSC}$, has a better acquisition frequency (average value $0.35 \mathrm{~s}$ ) and showed better quantification performances.

Table 2 summarizes the figures of merit obtained from calibration curves (up to $5 \mathrm{mg} \mathrm{L}^{-}$ $\left.{ }^{1}\right)$ using SIM MS acquisition mode. Good linear correlations were found $\left(r^{2}>0.98\right)$. The instrumental sensitivity was calculated as three times the standard deviation of the chromatographic noise from injecting blank solutions, being the minimum detectable amount values from 0.9 (dimethyl disulfide) to $180 \mathrm{ng} \mathrm{L}^{-1}$ (hydrogen sulfide). Quantification limit was calculated as ten times the standard deviation of the chromatographic noise from injecting blank solutions. The values, ranged from 3 225 (dimethyl disulfide) to $364 \mathrm{ng} \mathrm{L}^{-1}$ (hydrogen sulfide). Reproducibility, expressed as relative standard deviation (five replicates), was lower than $6 \%$. In conclusion, the 
analytical performances were adequate for the analysis of organic solutions containing RSC at trace levels.

\section{Sampling and extraction conditions}

230 An efficient sampling of atmospheric volatile compounds depends on the sampling support and the later extraction procedure. Particularly, the determination of volatile RSCs has several important difficulties, including their low range of concentrations and their highly reactive nature. According to our previous experiments [12], activated coconut charcoal sorbents are excellent materials for trapping $\mathrm{CS}_{2}$ by an adsorption

235 mechanism. In the present research, two solid-phase supports (Orbo-32 and Anasorb CSC) were used and compared to Tenax, a solid-supports previously described for capturing RSCs [6]. The proposed cartridges are extremely porous materials, with a large surface to volume ratio and a small diffusion distance. Concretely, Orbo-32 support has a mesh size of 20-40 and surface area of $300 \mathrm{~m}^{2} \mathrm{~g}^{-1}$ and Anasorb CSC

240 support has a mesh size of $20-40$ and surface area of $980 \mathrm{~m}^{2} \mathrm{~g}^{-1}$, compared to Tenax with $60-80$ and $35 \mathrm{~m}^{2} \mathrm{~g}^{-1}$, respectively.

Sampling efficiency was evaluated by pumping air mixtures of RSCs and determining their concentration in the outlet stream. Experimental data indicated that the proposed supports enabled the effective collection of target molecules.

245 For recovering targeted pollutants from tested trapping materials, solvent desorption was studied. The best protocol was sampling with Anasorb CSC supports followed by hexane as extraction solvent at extraction volume of $2 \mathrm{~mL}$. The analysis of blank air samples demonstrated that no contamination occurred, yielding clean chromatograms. The select method also avoided overlapping peaks between RSCs and method reagents, e.g. solvent residues. Table 3 shows the recovery percentages for extraction step, of both types of supports employing hexane, toluene and methanol as solvent extractor. The results under the best protocol extraction - SKC support and hexane as solvent - were hydrogen sulfide (71.4\%), ethyl mercaptan (93\%), dimethyl sulfide (85\%), carbon disulfide (95\%), butyl mercaptan (75\%) and dimethyl disulfide (97\%) respectively, reaching a mean recovery of $86 \%$. This method was compared to sampling with TENAX cartridge and thermal desorption. The recoveries ranged from $55.5 \%$ (hydrogen sulfide) to $81.8 \%$ (carbonyl disulfide), respectively. Therefore, significant differences were observed between both methods (paired t-test, $p$ value $<0.01)$. The values using coconut charcoal sorbents were similar better to those 260 previously reported using other solid-supports [12-15]. The loss patterns of RSCs depended on interaction with column substrate, etc... Moreover, lighter and high reactivity RSCs can be also subject to less efficient recovery (Pandey et al., 2009). 
The breakthrough volume was determined by studying the release of RSC compounds in the outlet stream due to an excessive sampling volume. For that, air passed (volume up to $4.3 \mathrm{~m}^{3}$ ) through spiked cartridges and the malodor compounds were determined, calculating recovery percentages in both beds of sampling cartridges. Since breakthrough depends mainly on the total air volume of sample passed, tests were carried out at a fixed sampling air flow of $1 \mathrm{~L} \mathrm{~min}^{-1}$. Recovery percentages were obtained by testing the RSCs gas compounds using EUPHORE photo-reactor for

270 calibration. The results were totally satisfactory since RSCs were completely recovered For a maximum of $72 \mathrm{~h}$-period (total air volume up to $4.3 \mathrm{~m}^{3}$ ), the breakthrough corresponded an average RSC compounds concentration approximately 100 times higher than landfill environmental concentrations (ranged between 1-30 $\mathrm{mg} \mathrm{m}^{-3}$ depend of RSC compounds). EU legislation has set only a limit of $10 \mu \mathrm{g} \mathrm{m}^{-3}$ of carbon disulfide

275 - average concentration in $24 \mathrm{~h}-$ and the levels allowed by U.S.A. legislation are less than $10 \mu \mathrm{g} \mathrm{m}^{-3}$ - average concentration in $8 \mathrm{~h}$. The results were satisfactory, because RSCs were completely recovered from the top layer of the solid-phase sorbent (test t: $p$-value $>0.05$ ).

Other problems in the sampling of malodor volatile compounds in ambient air, including 280 RSCs, are the humidity effect and the interference caused by atmospheric oxidants [6]. Water may cause baseline perturbations and retention time shifts in the chromatographic separation, meanwhile oxidants, such as $\mathrm{SO}_{2}, \mathrm{O}_{3}$, and $\mathrm{NO}_{\mathrm{x}}$, can degrade the collected molecules. Experiments were designed to evaluate both effects generating air mixtures with RSCs compounds at $100 \mu \mathrm{g} \mathrm{m}^{-3}$, a relative humidity from 0 285 to $60 \%, \mathrm{O}_{3}$ at $200 \mu \mathrm{g} \mathrm{m}^{-3}$ and, $\mathrm{NO}_{x}$ at $500 \mu \mathrm{g} \mathrm{m} \mathrm{m}^{-3}$. Quantitative recoveries were obtained, being the values comparable to control experiments (test $t: p$-value>0.01). The stability of the RSC malodor compounds on coconut charcoal sampling cartridges was assessed to guarantee applicability for field atmospheric monitoring. For that, their concentrations were determined after storage at two conditions. The short-term storage 290 study shows that RSCs had important losses after 5 days at $4{ }^{\circ} \mathrm{C}$ (Figure 2). However, the losses were lower than $10 \%$ for time intervals up to 2 days (t-test, $p>0.05$ ). The long-term storage study indicated no changes were detected for samples stored for a time interval of 3 months at $-20^{\circ} \mathrm{C}$ (test $\mathrm{t}$ : $\mathrm{p}$-value>0.05). These results were better than the obtained by sampling methods based on Tedlar Bags [12], Tenax TA cartridges 295 [15], Carbotrap, Carbopack X and Carboxen 569 cartridges [2] and SPME [13]. These approaches require short storage of sampling supports; thereby the sample analysis must carried out before $24 \mathrm{~h}$ to avoid losses of RSC. Thus, the proposed method based on activated coconut charcoal cartridges overcome the current monitoring techniques of sulfur malodor compounds. 


\section{Figures of merit}

The analysis of spiked samples prepared by serial dilution in pure air provided the calibration curves, including all steps of the analytical procedure. Good linear correlations were found $\left(r^{2}>0.98\right)$. Table 4 lists the detection and quantification limits.

305 The detection limits ranged between $0.6 \mathrm{pg} \mathrm{m}^{-3}$ for dimethyl disulfide and $87 \mathrm{pg} \mathrm{m}^{-3}$ for hydrogen sulfide, that corresponded to $0.4-65$ pg (or $0.2-23 \mathrm{ppqV}$ ) considering the sampled air volume. These values were quite better - in three magnitude orders - than obtained by silica gel as solid sorbent sampling and detection by mobile mass spectrometer with atmospheric pressure chemical ionization [20]. Similar improvement was achieved compared to the values reported for samples collected by canisters and analyzed by GC-MS [8]. Our values were similar than those using cryofocusing sampling system coupled to a thermal desorption, analyzed by GC-PFPD (pulsed flame photometric detector) [16,19,21].

Replicate analysis, from ten fractions of an air sample at $0.1 \mathrm{mg} \mathrm{m}^{-1}$ of selected

315 compounds, confirmed the good precision of the proposed method (Table 4). The relative intra-day reproducibility ranged between 3 and $16 \%$ (mean 9\%) and the relative inter-day reproducibility ranged between 10 and $25 \%$ (mean 17\%). These values were similar than previously reported $[2,13]$.

Compared to the previous methods based on GC-MS, the analysis time in an important advantage [12,19]. Our results were provided in less than $30 \mathrm{~min}$, being $20 \mathrm{~min}$ for extraction and $10 \mathrm{~min}$ for GC-MS analysis. Only on-site instruments such as electronic nose devices provided faster responses ( $3 \mathrm{~min}$ ), but with very limited reproducibility, working range, and sensitivity (several magnitude orders) [20].

The analysis of gaseous mixtures, containing RSCs (from $5 \mu \mathrm{g} \mathrm{m}^{-3}$ to $1 \mathrm{mg} \mathrm{m}^{-3}$ ), showed the absence of false-positive and false-negative results. The mean errors were lower than 9\%, demonstrating the extended working range and accuracy of the proposed methodology for quantitative determination.

The proposed method was validated by analyzing air samples collected in control areas free of RSCs (Control 1 and Control 2). Then, the standard addition method was applied to estimate the presence of proportional systematic error. No interferences or alterations appeared along the registered chromatograms. The slope obtained in the standard addition method (peak area) agreed with the slope of the calibration curve (test $\mathrm{t}: \mathrm{p}$-value>0.05). Thus, the results indicated the absence of matrix effect, i.e. systematic errors, in the determination of RSCs, demonstrating the reliability of the developed method. 


\section{Analysis of environmental samples}

The methodology was applied to ambient air samples collected at three different areas close to three landfills, which process high amounts of urban and industrial wastes. The emissions contained between 3 and 5 of the studied RSC compounds. The 4-carbon compound (butyl mercaptan) was no detected in any sample, and hydrogen sulfide and ethyl mercaptan were measured in all the samples. Significant differences on RSCs values were observed, but not between landfills. Figure 3 shows the order of concentration RSCs was hydrogen sulfide > ethyl mercaptan > carbon disulfide >

345 dimethyl sulfide > dimethyl disulfide. The mean concentration of the most abundant compounds (hydrogen sulfide and ethyl mercaptan) were 608 and $493 \mathrm{gg} \mathrm{m}^{-3}$, being the maximum concentrations 1950 and $3066 \mu \mathrm{g} \mathrm{m}^{-3}$, respectively. The rest of detected RSC malodor compounds were present at considerably low concentrations, being the differences up to 2-orders. Their average values were $139 \mu \mathrm{g} \mathrm{m}^{-3}$ (carbon disulfide), 41 $350 \mu \mathrm{g} \mathrm{m}^{-3}$ (dimethyl disulfide), and $8 \mu \mathrm{g} \mathrm{m}^{-3}$ (dimethyl sulfide), and the maximum concentration values were $397 \mu \mathrm{g} \mathrm{m}^{-3}$, and $79 \mu \mathrm{g} \mathrm{m}^{-3}$ and, $19 \mu \mathrm{g} \mathrm{m}^{-3}$, respectively.

Similar concentration levels were reported in a landfill site, including municipal solid waste-related area, the leachate-related area and the sludge-related area [4]. Lower and higher concentrations were determined at landfills in China [2, 5]. Mostly, the data

355 could be explained due to the wide variability of the urban residues treated in these landfills [1]. Therefore, these results emphasize the need of methods that allow intensive sampling campaigns for these malodor sources compared to other industrial emissions, e.g. pulp industry [18].

The environmental implications of this sampling campaign were evaluated, calculating

360 the total reduced sulfur compounds (TRS), as the sum of concentrations of our determined RSC compounds, since any additional molecules containing sulfur atoms were identified in the chromatograms. According to the classification of air quality based on TRS thresholds, $60 \%$ of the samples were categorized as very poor air quality (TRS>1000 ppbV), indicating a severe malodor. In the rest of the cases, air 365 quality was poor (28>TRS>999 ppbV), indicating a strong malodor. A discussion is described based on the obtained results. First, the method provided fingerprint information, indicating which malodor compound was emitted and their concentration level. Hence, at this stage, the hypothesis that hydrogen sulfide and ethyl mercaptan are the substantial components of malodor phenomenon in the studied landfills could

370 be drawn. Second, the analytical performances of developed method are suitable for the control of environmental guidelines. This study showed that the carbon disulfide concentration levels exceeded European regulations in $100 \%$ of the cases studied 
(threshold level: $10 \mu \mathrm{g} \mathrm{m}^{-3}$ or $3.2 \mathrm{ppbV}$ ). This developed methodology is also useful for other application such as the implementation of specific mitigation actions, the confirmation of complains from the neighbors, among others.

\section{CONCLUSIONS}

A solid-phase sampling by activated charcoal surfaces sampling, hexane as an organic extraction solvent and separation by gas chromatography without derivation, and detection by mass spectrometry is an innovative approach. The simultaneous determination of several malodorous RSCs by a fast, sensitive, reproducible and efficient method improves the current state of the art. The proposed methodology supported an accurate malodor monitoring of located source processes and increasing the tolerance to sample storage between sampling and instrumental analysis.

This study, focused on the malodor emissions from landfills, reinforced the need for analytical tools for a systematic monitoring of RSCs, since their presence was detected in a high concentration levels and in a high number of samples. This fingerprint characterization of malodor pollution will help us to protect human and environmental health from possible adverse effects generated by the diverse human activities.

\section{ACKNOWLEDGMENTS}

The Instituto Universitario CEAM-UMH is partly supported by Generalitat Valenciana, and DESSESTRES (Prometeo - Generalitat Valenciana). We also acknowledged the European Community's Seventh Framework Program under the grant agreement no. 228335 (Eurochamp2), the Spanish Ministry of Science and Innovation, through INNPLANTA project: PCT-440000-2010-003 and the EUPHORE staff. The authors also thank A.T.B. and S.M. for their contribution.

\section{REFERENCES}

400 1. Muñoz, R., Sivret, E.C., Parcsi, G., Lebrero, R., Wang, X., Suffet, I.H., Stuetz, R.M. Monitoring techniques for odour abatement assessment. Water research 44 (2010), 5129-5149.

2. Kim, K.H. Some Insights into the Gas Chromatographic Determination of Reduced Sulfur Compounds (RSCs) in Air. Environ. Sci. Technol. 39 (2005) 6765-6769.

3. Trabue, S., Scoggin, K., Mitloehner, F., Li, H., Burns, R., Xin, H. Field sampling method for quantifying volatile sulfur compounds from animal feeding operations. Atmos. Environ. 42 (2008) 3332-3341. 
4. Fang, J.J., Yang, N., Cen, D.Y., Shao, L.M., He, P.J. (2012). Odor compounds from different sources of landfill: Characterization and source identification. Waste management, 32(7), 1401-1410.

5. Duan, Z., Lu, W., Li, D., Wang, H. 2014. Temporal variation of trace compound emission on the working surface of a landfill in Beijing, China. Atmos. Environ, 88, 230-238.

415 6. Pandey, S.K., Kim, K.H. A Review of Methods for the Determination of Reduced Sulfur Compounds (RSCs) in Air. Environ. Sci. Technol. 43 (2009) 3020-3029.

7. Higgins, M.J., Chen, Y.C., Yarosz, D.P., Murthy, S.N., Maas, N.A., Glindemann, D., Novak, J.T. Cycling of volatile organic sulfur compounds in anaerobically digested biosolids and its implications for odors. Water environment research, (2006), 243-252.

8. Guo, H., Simpson, I.J., Ding, A.J., Wang, T., Saunders, S.M., Wang, T.J., Cheng, H.R., Barletta, B., Meinardi, S., Blake, D.R., Rowland, F.S. Carbonyl sulphide, dimethyl sulfide and carbon disulfide in the Pearl River Delta of southern China: Impact of anthropogenic and biogenic sources. Atmos. Environ. 44 (2010) 3805-3813.

9. Kabir, E., Kim, K. H. Use of solid phase microextraction (SPME) in the analysis of the reduced sulfur compounds (RSC) and its experimental limitations. Microchemical Journal, 103, (2012) 42-48.

10. Zhao, P., Liu, J., Wang, L., Yi, Q. Novel multi-sorbent for sampling and determination of trace volatile organic sulfur compounds in ambient air. International J.Environ. Anal. Chem., 93(1), (2013), 48-60.

11. Davoli, E., Gangai, M. L., Morselli, L., Tonelli, D. Characterisation of odorants emissions from landfills by SPME and GC/MS. Chemosphere, 51(5), ,357-368.

12. Kim, K.H., Choi, Y.J., Oh, S.I., Sa, J.H., Jeon, E.C., Koo, Y.S. Short-term 435 distributions of reduced sulfur compounds in the ambient air surrounding a large landfill facility. Environ Monit Assess 121 (2006) 343-354.

13. Nielsen, A.T., Jonsson, S. Quantification of volatile sulfur compounds in complex gaseous matrices by solid-phase microextraction. J. Chrom. A, 963 (2002) 57-64.

440 14. Campos, V.P., Oliveira, A.S., Cruz, L.S.C., Borges, J., Tavares, T.M. Optimization of parameters of sampling and determination of reduced sulfur compounds using cryogenic capture and gas chromatography in tropical urban atmosphere. Microchem. J. 96 (2010) 283-289. 
15. Ras, M. R., Marcé, R. M., Borrull, F. Solid-phase microextraction-Gas chromatography to determine volatile organic sulfur compounds in the air at sewage treatment plants. Talanta, 77(2), (2008) 774-778.

16. Catalan, L.J.J., Liang, V., Jia, C.Q. Comparison of various detection limit estimates for volatile sulphur compounds by gas chromatography with pulsed flame photometric detection. J. Chrom A, 1136 (2006) 89-98.

17. Deshmukh, S., Jana, A., Bhattacharyya, N., Bandyopadhyay, R., R.A. Pandey. Quantitative determination of pulp and paper industry emissions and associated odor intensity in methyl mercaptan equivalent using electronic nose. Atmos. Environ. 82, (2014) 401-409.

18. Borrás, E., Ródenas, M., Dieguez, J.J., Pérez-García, M.L., Lomba, R., Lavín, J., Tortajada-Genaro, L.A. Development of a gas chromatography - mass spectrometry method for the determination of carbon disulfide in the atmosphere. Microchemical Journal 101, (2012) 37-42.

19. Susaya, J., Kim, K.H., Phan, N.T., Kim, J.C. Assessment of reduced sulfur compounds in ambient air as malodor components in an urban area. Atmos. Environ. 45 (20) (2011) 3381-3390.

20. Kudryavtsev, A.S., Makas, L.A., Troshkov, M.L., Grachev, M.A., Podyachev, S.P. The method for on-site determination of trace concentrations of methyl mercaptan and dimethyl sulfide in air using a mobile mass spectrometer with atmospheric pressure chemical ionization, combined with a fast enrichment/separation system. Talanta, 123, (2014) 140-145.

21. Kim, H.K. Consideration on the broad quantification range of gaseous reduced sulfur compounds with the combined application of gas chromatography and thermal desorber. Atmos. Enviro., (2011) 381-386.

470

475 


\section{TABLE CAPTIONS}

Table 1. A brief description of the reduced sulfur compounds investigated in this study.

Table 2. Analytical performances of GC-MS methodology in SIM mode $(n=10)$.

Table 3. Recovery percentages sampling by coconut charcoal sorbents and solvent 495 extraction $(n=5)$.

Table 4. Method detection and quantification limits, reproducibility and percentage of recovery for RSC compound selected $(n=10)$.

500

\section{FIGURE CAPTIONS}

Figure 1. Chromatogram of a standard solution containing selected RSC compounds at $5 \mathrm{mg} \mathrm{L}^{-1}$ in hexane. Detection based on SIM mode, using selected $\mathrm{m} / \mathrm{z}$ fragments: $34,62,76,90,94$.

505

Figure 2. Normalized concentration of RSCs compounds at different storage days at 4 ${ }^{\circ} \mathrm{C}(\mathrm{n}=10)$. Initial concentration $=5 \mathrm{mg} \mathrm{L}^{-1}$. Student's test: * $\mathrm{p}$-value $<0.1$, ${ }^{* *} \mathrm{p}$ value $<0.01$.

510 Figure 3. Results of RSCs compounds determined from landfills (51 samples). 